\title{
Scenario of Mushroom Poisoning in a Tertiary Care Hospital
}

\author{
Syed VA, ${ }^{1}$ Lakshmi R, ${ }^{1}$ Karki P, $^{1}$ Pradhan B, ${ }^{1}$ Maskey R' \\ 'Department of Internal Medicine, BP Koirala Institute of Health Sciences, Dharan, Nepal.
}

\section{ABSTRACT}

Introduction: Mushroom poisoning is not new to Nepal. Undocumented and unrecorded mushroom poisoning is more common than the published one. We evaluated the pattern and outcomes following toxic mushroom ingestions.

Methods: We did a retrospective analysis of patients admitted with mushroom poisoning in BPKIHS, a tertiary care hospital in Nepal, from 2002 to 2006. The diagnosis of mushroom poisoning was based on history and clinical presentation.

Results: Twenty seven patients, aged between 7-83 years were studied. Fourteen $(51.86 \%)$ were male and $13(48.14 \%)$ were female. All $(100 \%)$ patients had consumed wild mushroom unintentionally. Twelve patients developed symptoms within first hour and 11 patients within one to six hours. The average delay in presenting to our hospital was 23.1(2-96) hours. In 10 cases, other family members were affected, out of which in two cases the family members had died in other hospitals. Reported symptoms were nausea $27(100 \%)$, vomiting 24 (88.88\%), abdominal cramping 8 (29.6\%), jaundice 3 $(11.1 \%)$, diarrhea $17(62.96 \%)$ and altered sensorium in 8 patients $(29.65 \%)$. All patients were treated conservatively. Three patients with severe hepatitis were admitted in ICU, out of which two recovered without liver transplant. One eight year old boy developed acute liver failure and expired within 24 hours of hospitalization.

Conclusions: Most of the mushroom poisoning was acute, unintentional and by consumption of wild mushroom. Community based awareness programs will help prevent the instances of poisoning.

Key Words: Nepal, mushroom poisoning, retrospective study

\section{INTRODUCTION}

The history of mushrooms is surrounded by mystery and superstition. This mystique is perhaps associated with the poisonous mushroom's reputation. Most mushroom species, however, are not poisonous; of the 5,000 known species of mushrooms, fewer than 100 are poisonous. ${ }^{1}$ Nepalese mushrooms are tabulated as follows, 110 edible,
Correspondence:

Dr. Prahlad Karki

Department of Internal Medicine

BPKIHS, Dharan, Nepal.

Email: svaqarahmad@yahoo.com

Phone: 9852045913 
13 medicinal, 45 toxic and 6 others. $^{2}$ Many Nepalese collect, eat and sell wild mushroom and mushroom poisoning is rarely reported here.

Poisonous mushrooms have no characteristic smell or taste and often resemble edible mushrooms. Unfortunately, edible and non edible mushrooms are frequently misidentified by mushroom collectors. ${ }^{3}$ Whereas severe mushroom poisonings are rare, about 100 to 200 fatalities per year due to mushroom poisoning occur in the United States and Europe. Moreover, $90 \%$ to $95 \%$ of deaths are caused by a species, Amanita phalloides. ${ }^{1}$ Patients poisoned with toxic mushrooms have a wide spectrum of clinical presentations. To optimize care, early diagnosis is essential.

\section{METHODS}

A retrospective study was conducted for demographical features, laboratory data, and applied treatment of the patients admitted with mushroom poisoning in BPKIHS, a tertiary care hospital in Nepal from January, 2002 to December, 2006. The ethical and institutional approvals were taken. The patients with only the mushroom poisoning were included. The diagnosis of mushroom poisoning was based on careful history (consumption of toxic mushrooms) taking primarily. None of the leftover mushroom was chemically analyzed, because chemical analysis was not done at our centre.

The initial laboratory evaluations were complete blood count, arterial blood gas, serum glucose, electrolytes, creatinine, urea and liver function tests [alanine (ALT), aspartate aminotransferase (AST), alkaline phosphatase and prothrombin time)]. The results were expressed as mean SD and were statistically analyzed by Microsoft excel 2003.

\section{RESULTS}

Of the 421 poisoning cases admitted to BPKIHS, only 27 were poisoned by consumption of toxic mushrooms. All 27 files were included in this study. There were 14 (51.1\%) male and $13(48.9 \%)$ female. The patient's age ranged between 7 and 83 years, with a mean of $27.26 \pm 19.96$ years. Eleven patients were less than 14 years of age. All cases of poisoning were unintentional and by the consumption of wild mushroom. The most common geographic site of exposure 27 (100\%) was a residence (Table 1 ).
Table. 1 Baseline Characteristics of the patients with mushroom poisoning

\begin{tabular}{ll} 
Characteristics of the patients & Results \\
\hline Number of patients & $<14 \mathrm{yrs}$ \\
$27(100 \%)$ & $11(44.4 \%)$ \\
Age (yrs) Mean $\pm \mathrm{SD}$ & $27.26 \pm 19.96$ \\
Sex (\%) M:F & $51.9: 48.1$ \\
First symptom after mushroom ingesting & \\
<6hrs & $23(85.2 \%)$ \\
>6hrs & $4(14.8 \%)$ \\
Delay in reaching our hospital: hrs range (mean) & $2-96(20.74)$ \\
Vomiting & $24(88.9 \%)$ \\
Jaundice & $3(11.1 \%)$ \\
Diarrhea & $17(63 \%)$ \\
Pain abdomen & $8(29.6 \%)$ \\
Alter sensorium & $13(48.1 \%)$ \\
ALT (U/L) & $120 \pm 343.7$ \\
AST (U/L) & $100.78 \pm 263.6$ \\
S.Alkaline Phosphatase (U/L) & $197.7 \pm 106$ \\
Prothrombin time (sec) & $22.3 \pm 36.2$ \\
T. Bilirubin (mg/dl) & $1.36 \pm 1.89$ \\
Urea (mg/dl) & $26.52 \pm 10.97$ \\
Creatinine (mg/dl) & $0.76 \pm 0.33$ \\
Duration of hospital stay (days) & $2.37 \pm 2.2(1$ to 12$)$ \\
ICU admission (\%) & $2(7.4 \%)$ \\
I.V.fluid & $100 \%$ \\
Lavage & $88.9 \%$ \\
Antibiotics & $3(11.1 \%)$ \\
Recovery (\%) & $26(96.3 \%)$ \\
Mortality (\%) & $1(3.7 \%)$ \\
\hline & \\
\hline
\end{tabular}

Most of poisoning cases were admitted from the month of April to August but maximum were in the month of June with a frequency of $59.2 \%$. Twelve patients developed symptoms within first hour, there were 5 male and 7 female with average age of 27 and 29.7 years respectively, 1 male and 2 female were less than 14 years and 11 patients developed symptoms within one to six hours, there were 6 male and 5 female with average age of 16.5 and 28.2 years respectively, five male and one female were less than 14 years. Four patients developed symptoms after six hours of eating mushroom.

The average delay in presenting to our hospital was 20.74 (2-96) hours. In 10 cases, other family members were affected, out of which two other family members, 10 years old male child and 55 years old female had died in other hospitals. The most frequently reported symptoms were vomiting 24 (88.9\%), nausea 25 (92.60\%), abdominal pain $8(29.60 \%)$ and diarrhea $17(63 \%)$. Alter sensorium was reported in $8(29.6 \%)$ patients. Jaundice was observed in $3(11.1 \%)$ cases (Table 2$)$. 
Table. 2 Characteristics of mushroom induced hepatitis

\begin{tabular}{ll}
\hline Characteristics & Results \\
\hline Number of patients & 3 \\
<14yrs & $2(66.67 \%)$ \\
\hline Age (yrs) Mean \pm SD & $17 . \pm 11.53$ \\
Sex M:F & $2: 1$ \\
First symptom after mushroom ingesting & \\
<6hrs & $1(33.3 \%)$ \\
>6hrs & $2(66.7 \%)$ \\
Delay in reaching our hospital: range (mean) & $4-72(25.41)$ hrs \\
Vomiting & $3(100 \%)$ \\
Jaundice & $3(100 \%)$ \\
Diarrhea & $3(100 \%)$ \\
Pain abdomen & $1(33.33 \%)$ \\
Alter sensorium & $3(100 \%)$ \\
ALT (U/L) & $903.7 \pm 706.44$ \\
AST (U/L) & $734 \pm 475.3$ \\
S.Alkaline Phosphatase (U/L) & $404 \pm 209$ \\
Prothrombin time (sec) & $99.33 \pm 92.5$ \\
T. Bilirubin (mg/dl) & $6.4 \pm 1.6$ \\
Urea (mg/dl) & $24.33 \pm 12.1$ \\
Creatinine (mg/dl) & $0.83 \pm 0.35$ \\
Duration of hospital stay (days) & $2.37 \pm 2.22(1$ to 12$)$ \\
ICU admission (\%) & $3(100 \%)$ \\
I.V.fluid & $3(100 \%)$ \\
Antibiotics & $3(100 \%)$ \\
Recovery (\%) & $2.6 \%)$ \\
Mortality (\%) & \\
\hline
\end{tabular}

Mean Prothrombin time (PT) at the time of presentation was $22.3 \pm 36.2 \mathrm{sec}$ (Table1). The PT was $99.33 \pm 92.5 \mathrm{sec}$ in patients with hepatitis. There were three patients with hepatitis, two of them improved without liver transplantation (Table 2).

Treatment included intravenous hydration (100\% of patients), $\mathrm{H}_{2}$ blockers (100\%), activated charcoal 24 (88.9\%), antibiotics $3(11.1 \%)$, and Fresh frozen plasma and vitamin $\mathrm{K}$ were administered to $1(3.7 \%)$ and $3(11.1 \%)$ patients, respectively. A total of $96.3 \%$ of patients were discharged and the duration of hospitalization was $2.37 \pm 2.22$ (1 to 12$)$ days.

Only $3(11.1 \%)$ patients were admitted to a critical care unit, and $24(88.8 \%)$ were admitted to non-critical care unit. Only one death was reported during the study period, and this occurred in an eight-year-old child who developed acute liver failure with multi organ dysfunction and required mechanical ventilation. He expired within 24 hours of admission.

\section{DISCUSSION}

No adequate database exists to estimate worldwide mushroom exposures. In the US, in 1996, 10,584 mushroom exposures were reported to the American Association of Poison Control Centers. Eighty-eight percent of reported mushroom exposures were unidentified. ${ }^{4}$ Only 54 were identified as amatoxin exposures, however, this number is undoubtedly an underestimation given the number of unknown mushroom exposures.

In our study, 27 cases of mushrooms poisoning, one of whom died, were admitted to BPKIHS. The majority of the patients consumed wild mushrooms and they had collected those from forest and fields without being aware of the dangers associated with eating those.

The gathering and consumption of wild mushrooms is a traditional social outing in many countries. This habit is particularly widespread in many European; American and Asian countries. ${ }^{4}$ ${ }^{8}$ Considerable differences in symptoms may be seen with various mushroom species due to seasonal and location variance in the toxin content, variance in oral bioavailability, differences in metabolic activation, and differences in toxin susceptibility. ${ }^{9}$ Reporting of mushroom poisoning is rarely done in Nepal. We do not know, what is the exact burden of poisoning here?

Toxic mushroom poisonings lead to a variety of clinical outcomes ranging from self-limited gastrointestinal symptoms to fulminant hepatic failure requiring orthotopic liver transplantation. Fourteen major syndromes of mushroom poisoning are: first by presentation timing and then by target organ systemic toxicity and included early ( $<6 \mathrm{hrs}$ ), late (6-24 hrs), and delayed syndromes (more than or equal one day). There were eight early syndromes (four neurotoxic, two gastrointestinal, two allergic); three late syndromes (hepatotoxic, accelerated nephrotoxic, erythromelalgia); and three delayed syndromes (delayed nephrotoxic, delayed neurotoxic, rhabdomyolysis). Four new mushroom poisoning syndromes were classified including accelerated nephrotoxicity (Amanita proxima, Amanita smithiana), rhabdomyolysis (Tricholoma equestre, Russula subnigricans), erythromelalgia (Clitocybe amoenolens, Clitocybe acromelalgia), and delayed neurotoxicity (Hapalopilus rutilans). ${ }^{10}$

Each mushroom group exerts its toxic effect by a different mechanism, and certain toxins have a predilection for individual organ systems. The amatoxins (cyclic octapeptides), which include amanitin, verotoxin, and phalloides, cause severe hepatocellular damage by inhibiting RNA polymerase II, thereby inhibiting protein synthesis at the cellular level, causing cell death. Other organ systems with high turnover rates (eg, gastrointestinal tract, kidneys) also are affected severely. Ibotenic acid and muscimol bind to glutamic acid and GABA receptors, respectively, and thereby interfere with CNS receptors. Monomethylhydrazine ( $\mathrm{MMH}$ ) from gyromitrin-containing

\section{JNMA | Val 48}


mushrooms affects the GI tract, liver, and kidneys by inhibiting pyridoxine-dependent pathways in the synthesis of GABA. Muscarine affects the autonomic nervous system through depolarization of muscarinic acetylcholine receptors and exerts a peripheral cholinergic effect through stimulation of the postganglionic parasympathetic receptors. Coprine inhibits aldehyde dehydrogenase, producing a disulfiramlike reaction in those consuming ethyl alcohol. Psilocybin indole exerts its effect on the central nervous system by stimulation of serotonin receptors. Orellanine and orelline, the bipyridyl toxins isolated from Cortinarius orellanus, exhibit their nephrotoxic effects by inhibiting alkaline phosphatase of the proximal tubule cells. Genetic factors may contribute to the clinical manifestations of this toxin, which has toxicity that is not reduced by cooking or drying. ${ }^{11}$ The most common symptoms of mushroom poisoning worldwide is a syndrome of malaise, epigastric pain, nausea, vomiting, and diarrhea that occurs within 30 minutes to three hours of ingesting a wide variety of inedible mushrooms and even some edible mushrooms, especially those that have recently decomposed in the field or in storage. Gastrointestinal toxin-containing mushrooms may come from several genera including Boletus, Chlorophyllum, Entoloma, Lactarius, Omphalotus, and Tricholoma. ${ }^{11}$

Most of our patients had gastrointestinal symptoms and $85 \%$ of the pateints developed symptoms with in first six hours. In a study done in California during December 1996 to January 1997, a case-patient was defined as a person with vomiting and diarrhea beginning six hours after consuming wild mushrooms. The median interval from ingestion to the onset of gastrointestinal symptoms was 12 hours (range 8-26 hours). Reported symptoms included vomiting (100\%), diarrhea $(100 \%)$, nausea $(100 \%)$, abdominal cramping $(60 \%)$, and weakness (70\%). ${ }^{1}$ Nine patients ( 8 male and 1 female) aged between 12 to 68 years were hospitalized for a median of 6 days (range 2-8 days); the two deaths, both male occurred on the sixth and eighth hospital days The most frequently reported symptoms in our case were vomiting $24(88.9 \%)$, nausea 25 (92.60\%), abdominal pain $8(29.60 \%)$ and diarrhea 17 (63\%). Alter sensorium was reported in $8(29.6 \%)$ patients. Jaundice was observed in $3(11.1 \%)$ of cases.

In a similar retrospective study of mushroom poisioning done in Iran, the most frequently reported symptoms were vomiting $(84 \%)$, nausea $(60 \%)$, abdominal pain $(60 \%)$ and diarrhea $(40 \%)$. Jaundice was observed in $44 \%$ of cases, with a $50 \%$ rate of hepatic encephalopathy. A total of $66 \%$ of patients were discharged and the duration of hospitalization was 1-12 days. ${ }^{12}$

Other mushroom species may produce toxic symptoms; these has been reviewed previously. ${ }^{9-11}$ The most dramatic effect of poisonous mushrooms is on the liver. Hepatotoxicity has been described following ingestion of the Gyromitra esulanta which contains gyromitrin which is hydrolyzed to monomethylhydrazine.The onset and character of symptoms are similar to amatoxin posioining. Amatoxins are actively absorbed and are extremely potent hepatotoxins. Amanitine is the principal toxin in this group. ${ }^{13} \mathrm{~A}$ considerable part of amatoxin is taken up by hepatocytes, excreted into the bile, and reabsorbed by entherohepatic cycle. ${ }^{14-17}$ There they are bound to RNA polymerase II and thus inhibit the formation of m-RNA. ${ }^{18}$ As a result of this, AST and ALT display a sharp increase. ${ }^{19}$

Hepatotoxic effect related to Ganoderma lucidum (Lingzhi) mushroom powder was first described in a patient from Hong Kong in 2004. In 2005, the authors experienced a case of fatal fulminant hepatitis associated with such a preparation. ${ }^{20}$ Recently in a retrospective study of the clinical classification of 3638 cases of mushroom poisoning in china from 1995 to 2004; the most common symptoms were those of acute renal failure in 1450 cases; among them 36 died (2.5\%). Symptoms of toxic hepatitis were found in 1010 cases, among them and 169 died $(16.7 \%){ }^{21}$

As with all poisonings, supportive care is of primary importance in mushroom poisioning. Airway management and cardiopulmonary resuscitation, along with maintenance of vital signs, should take priority over identification of the toxin and concern about antidotes. The greatest concern is about the ingestion of deadly Amanita species. Many antidotes and treatments have been suggested for this kind of poisoning, most of them unproven. If vomiting and diarrhea are severe enough to result in significant fluid loss, intravenous replacement of volume and electrolytes is important. The intravenous solution should contain glucose. Late syndromes (symptom onset $>6$ hrs after ingestion) are lifethreatening due to liver- and renal failure. Patients, who are jaundiced after an acute gastrointestinal episode, are suspected to be poisoned with Amatoxins. Patients with flank pain, hematuria, polyuria or oliguria in the absence of jaundice are suspected to have intoxication with Cortinarius mushrooms. In both cases an intensive care management is indicated.

Treatment for mushroom poisioning includes intravenous hydration (100\% of patients), $\mathrm{H}_{2}$-blockers (100\%), activated charcoal $(90 \%)$, penicillin $(60 \%)$, and $\mathrm{N}$-acetylcysteine $(80 \%)$. Fresh frozen plasma and vitamin $\mathrm{K}$ were administered to $30 \%$ and $60 \%$ of patients, respectively. ${ }^{1}$ In our study Six patients received repeated doses of activated charcoal. Other treatment included intravenous hydration (100\% of patients), $\mathrm{H}_{2}$-blockers (100\%), activated charcoal $24(88.9 \%)$, antibiotics $3(11.1 \%)$, and Fresh frozen plasma and vitamin $\mathrm{K}$ were administered to $1(3.7 \%)$ and $3(11.1 \%)$ of patients, respectively. 
Fatalities have been correlated with young age ( $<10$ years), short latency from ingestion to onset of gastrointestinal symptoms, and the severity of coagulopathy. ${ }^{13,14}$ In our case series only $3(11.1 \%)$ patients with were admitted to a critical care unit, and $24(88.8 \%)$ were admitted to the non-critical care unit. Only one death was reported during the study period, and this occurred in an eight-year-old child who developed acute liver failure with multi organ dysfunction, required mechanical ventilation. Our study is limited by the small number of retrospective cases. Future research in this area should focus on prospectively collecting data and pooling results among several centers to obtain more meaningful numbers.

\section{CONCLUSIONS}

Most of the mushroom poisonings were acute, unintentional and by consumption of wild mushroom. In all the instances the patients were unaware of the adverse effects of wild mushroom ingestion. Community based awareness programs will help to prevent the instances of poisoning. In conclusion, people should be more informed of the dangers posed by wild mushrooms. Training of physicians and nurses in the accurate diagnosis and management of patients poisoned with poisonous mushrooms would improve the rate of survival. A widespread campaign to inform people of the possible dangers of eating wild mushrooms would be useful.

\section{REFERENCES}

1. Yamada EG, Mohle-Boetani J, Olson KR, Werner SB. Mushroom poisoning due to amatoxin-Northern California, winter 19961997. West J Med. 1998;169:380-4.

2. Adhikari MK, Devkota S,Tiwari RD. Ethnomycological knowledge on uses of wild mushrooms in western and central Nepal. Our Nature. 2005; 3:13-9.

3. Olson KR, Pond SM, Seward J, et al. Amanita phalloides-type mushroom poisoning. West J Med. 1982 Oct;137:282-9.

4. Litovitz TL, Smilkstein M, Felberg L, et al. 1996 annual report of the American Association of Poison Control Centers Toxic Exposure Surveillance System. Am J Emerg Med. 1997 Sep; 15(5): 447-500.

5. Yilmaz A, Gursoy S, Varol O, Nur N, Ozyilkan E. Emergency room cases of mushroom poisoning. Saudi Med J. 2006 Jun; 27(6):858-61.

6. Pajoumand A, Shadnia S, Efricheh H, Mandegary A, HassanianMoghadam H, Abdollahi M. A retrospective study of mushroom poisoning in Iran. Hum Exp Toxicol. 2005 Dec; 24(12):609-13.

7. Bickel M, Ditting T, Watz H, Roesler A, Weidauer S, Jacobi V, et al. Severe rhabdomyolysis, acute renal failure and posterior encephalopathy after 'magic mushroom' abuse. Eur J Emerg Med. 2005 Dec; 12(6):306-8.

8. Unluoglu I, Tayfur M. Mushroom poisoning: an analysis of the data between 1996 and 2000. Eur J Emerg Med. 2003 Mar;10(1):23-6.

9. Koppel C. Clinical symptomatology and management of mushroom poisoning. Toxicon. 1993;31:1513-40.

10. Diaz JH. The epidemiology, toxidromic classification, general management, and prevention of mushroom poisoning in the United States. J La State Med Soc. 2005 Nov-Dec;157(6):330-6.

11. Stenklyft PH, Augenstein WL. Chlorophyllum molybdites: severe mushroom poisoning in a child. J Toxicol Clin Toxicol. 1990; 28:159-68.
12. Pajoumand A, Shadnia S, Efricheh H, Mandegary A, HassanianMoghadam H, Abdollahi M. A retrospective study of mushroom poisoning in Iran. Hum Exp Toxicology. 2005 Dec;24(12):60913.

13. Benjamin D. Mushrooms: Poisons and Panaceas. New York: W.H. Freeman and Company;1995.

14. O'Brien BL, Khuu L. A fatal sunday brunch: Amanita mushroom poisoning in a Gulf Coast family. Am J Gastroenterol. 1996; 91:581- 3.

15. Langer M, Gridelli B, Piccolo G, Markovic S, Quarenghi E, Gatti $\mathrm{S}$, et al. A liver transplant candidate (fulminant hepatic failure from Amanita phalloides poisoning) as a multiorgan donor. Transp Proc. 1997;29:3343-4.

16. Parra S, Garcia J, Martinez P, De la Pena C, Carrascosa C. Profile of the alkaline phosphatase isoenzymes in ten patients poisoned by mushrooms of the genus Lepiota. Dig Dis Sci. 1992;37:1495-8.

17. Cole FM. A puppy death and Amanita phalloides. Aust Vet Assoc.1993;70:271-2.

18. Kroncke KD, Fricert G, Meier PJ, Gerok W, Wieland T, Kurz G.Alpha-amanitin into hepatocytes. J. Biol Chem. 1986;261:12562-7.

19. Kendrick B, Shimizu A. Mushroom poisoning - analysis of two cases, and a possible new treatment, plasmapheresis. Mycologia. 1984;76:448-53.

20. Wanmuang H, Leopairut J, Kositchaiwat C, Wananukul W, Bunyaratvej S . Fatal fulminant hepatitis associated with Ganoderma lucidum (Lingzhi) mushroom powder. J Med Assoc Thai. 2007 Jan;90(1):179-80.

21. Ren CS, Wang WQ, Xu ZH, Lu HH, Gao QJ, Guo ZJ, et al. A study of the clinical classification of 3638 cases of mushroom poisoning. Zhonghua Nei Ke Za Zhi. 2007 Mar; 46(3):229-32. 